\title{
Microwave absorption in the frustrated ferrimagnet $\mathrm{Cu}_{2} \mathrm{OSeO}_{3}$
}

\author{
M.I. Kobets, K.G. Dergachev, E.N. Khatsko, and A.I. Rykova \\ B.Verkin Institute for Low Temperature Physics and Engineering National Academy of Sciences of Ukraine, \\ 47 Lenin Ave., Kharkov 61103, Ukraine \\ E-mail:khatsko@ilt.kharkov.ua \\ P. Lemmens and D. Wulferding \\ Institute for Condensed Matter Physics, TU Braunschweig, D-38106 Braunschweig, Germany \\ H. Berger \\ Institute de Physique de la Matiere Complexe, EPFL, CH-1015 Lausanne, Switzerland
}

Received August 27, 2009

\begin{abstract}
The resonance properties of a new $\mathrm{Cu}_{2} \mathrm{OSeO}_{3}$ ferrimagnet have been investigated in a wide range of frequencies (17-142 GHz) at liquid helium temperature. The resonance data were used to plot the frequencyfield dependence of the ferrimagnetic spectrum described within the model of an anisotropic two-sublattice ferrimagnet. The effective magnetic anisotropy corresponding to the gap in the spin wave spectrum has been estimated $(3 \mathrm{GHz})$. It is found that the spectrum has a multicomponent structure which is due to the diversity of the types of magnetization precession. As the amplitude of the high-frequency magnetic field increased, an additional absorption was observed in the external magnetic field lower than the field of the main resonance. The detected additional absorption corresponds to the nonuniform nonlinear parametric resonance, connected with nonuniformity of magnetic structure in the ferrimagnetic crystal $\mathrm{Cu}_{2} \mathrm{OSeO}_{3}$.
\end{abstract}

PACS: 76.50.+g Ferromagnetic, antiferromagnetic, and ferrimagnetic resonances; spin-wave resonance;

75.50.Ee Antiferromagnetics.

Keywords: ferrimagnet materials, frequency-field dependence, magnetization precession.

\section{Introduction}

Ferrimagnetic materials have a great importance in present-day physics and engineering, which is due to their widespread use in ultrahigh radio frequency technologies. Most of today's known ferrimagnets are nonmetallic compounds. The exchange interaction in ferrimagnets, like in antiferromagnets, imposes an antiparallel orientation on the moments belonging to different sublattices. A joint feature of these materials is the dissimilarity of their sublattices: their magnetization varies in magnitude generating spontaneous ferrimagnetic moments. Such variations are possible either because the sublattices contain different numbers of ions or because these ions have different moments. Ferrimagnets are the most suitable objects to investigate magnetic oscillations in the magnetically ordered state. They form a unique class of magnetic substances offering major advantages in microwave technologies.
The high-frequency (hf) properties of ferrimagnets are actually equivalent to the same properties of ferromagnets. In magnetically ordered crystals with any number of sublattices and a spontaneous exchange-type moment the spectrum of magnetic oscillations always contains one «ferromagnetic branch» which is independent of the exchange forces (molecular field) [1]. The specific character of a non-compensated antiferromagnet manifests itself as a second «exchange» resonance. Its eigenfrequencies now are dependent on the molecular field. They belong to the far IR region and are too difficult to observe. Many ferrimagnets exhibit a narrow resonance line and are suitable to investigate instability related resonance and nonlinear phenomena.

The aim of this study is investigation of resonant properties of a new ferrimagnetically ordered $\mathrm{Cu}_{2} \mathrm{OSeO}_{3}$ compound at liquid helium temperature.

The crystal $\mathrm{Cu}_{2} \mathrm{OSeO}_{3}$ is a magneto-capacitive system described by the cubic spatial symmetry group $P 2{ }_{1} 3$ with 
the lattice parameter $a=8.923 \AA[2]$ at room temperature. Magnetic $\mathrm{Cu}^{2+}$ ions, arranged tetrahedrally along the space diagonal [111], are the main element of the crystal structure, which complies with the general concept of ferrimagnetic compounds [3]. It is found [3] that cubic single crystals with negative anisotropy constant $K_{1}$ have directions of hard magnetization along the cubic cell edges and easy magnetization along the space diagonals of the cube. Fragments of the crystalline and magnetic structures of $\mathrm{Cu}_{2} \mathrm{OSeO}_{3}$ (taken from [2]) are shown in Figs. 1 and 2. It is seen that the oxygen atoms form square-pyramids as well as bi-pyramids around the tetrahedral arranged $\mathrm{Cu}^{2+}$. In $\mathrm{Cu}_{2} \mathrm{OSeO}_{3}$ the ground state is a non-compensated antiferromagnet (three spins are in the same direction and one spin is opposite). Geometrically, the compound has a

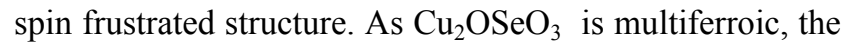
magnetoelectric coupling effects may play significant role in its behavior.

Resonance absorption was observed using a tunedfrequency radio spectrometer with different types of resonators (cylindrical and rectangular), each operating in its particular frequency range. The resonators were designed to include a device rotating the sample about the axis perpendicular to the applied permanent magnetic field $\mathbf{H}$. The microwave field $\mathbf{h}$ was polarized perpendicular to the applied magnetic field.

\section{Experimental results and discussion}

The resonance properties of $\mathrm{Cu}_{2} \mathrm{OSeO}_{3}$ were measured thoroughly in the frequency interval of $17-142 \mathrm{GHz}$ in magnetic fields up to $75 \mathrm{kOe}$ at liquid helium temperature. The size of the samples was smaller than the resonance wavelength. The samples were situated in a homogeneous ac magnetic field. The experimental width of the principal resonance line of the ferrimagnetic resonance in $\mathrm{Cu}_{2} \mathrm{OSeO}_{3}$ is $\Delta H_{0} \leq 18$ Oe (the real one is much smaller because we see already broadened lines) in the orientation $\mathbf{H} \| \mathbf{l}$.

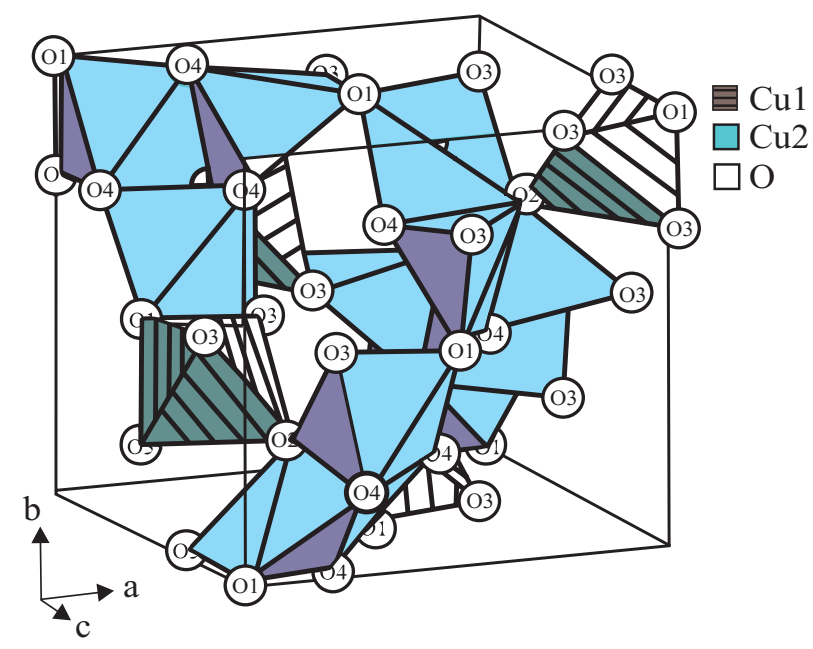

Puc. 1. A fragment of the crystal structure of single-crystal $\mathrm{Cu}_{2} \mathrm{OSeO}_{3}$.

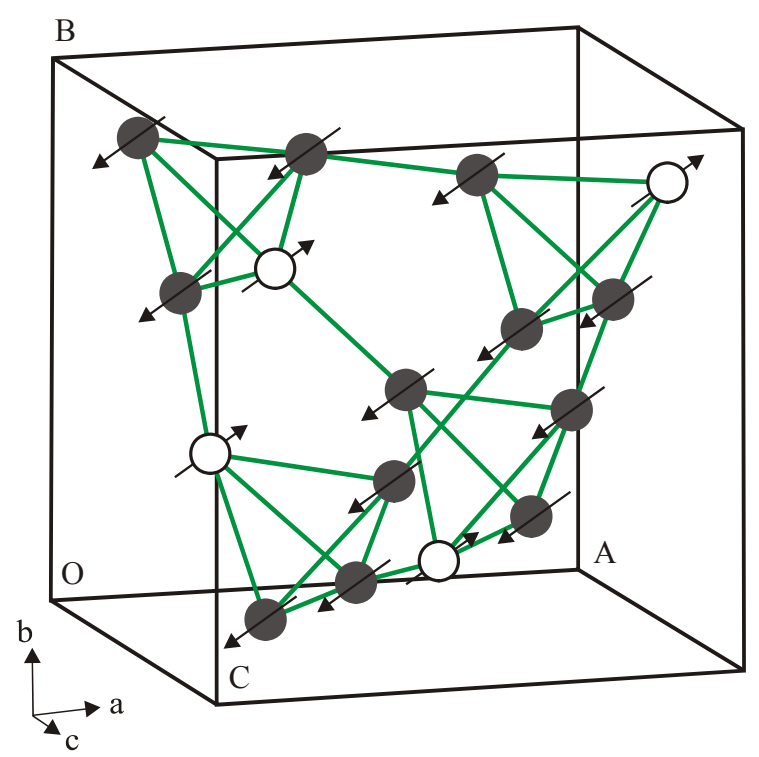

Puc. 2. The magnetic structure of single-crystal $\mathrm{Cu}_{2} \mathrm{OSeO}_{3}$.

The experimental frequency-field dependencies (measured at the low pumping power $P \sim 100 \mu \mathrm{W}$ ) of the ferrimagnetic spectrum along the easy axis ([111]) are shown in Fig. 3. It is seen that the experimental dependencies of the resonance spectrum do not coincide with the theoretical interpretation of low-frequency oscillations for a twosublattice ferrimagnet and cannot be described by the simple equation obtained for isotropic ferrimagnets in relatively low magnetic fields (smaller than an exchange field):

$$
\omega=\gamma_{\mathrm{eff}} H_{0} .
$$

The frequency-field dependences (Fig. 3) taken in a zero magnetic field have a gap of $3 \mathrm{GHz}(1 \mathrm{kOe})$ in the spin-wave spectrum.

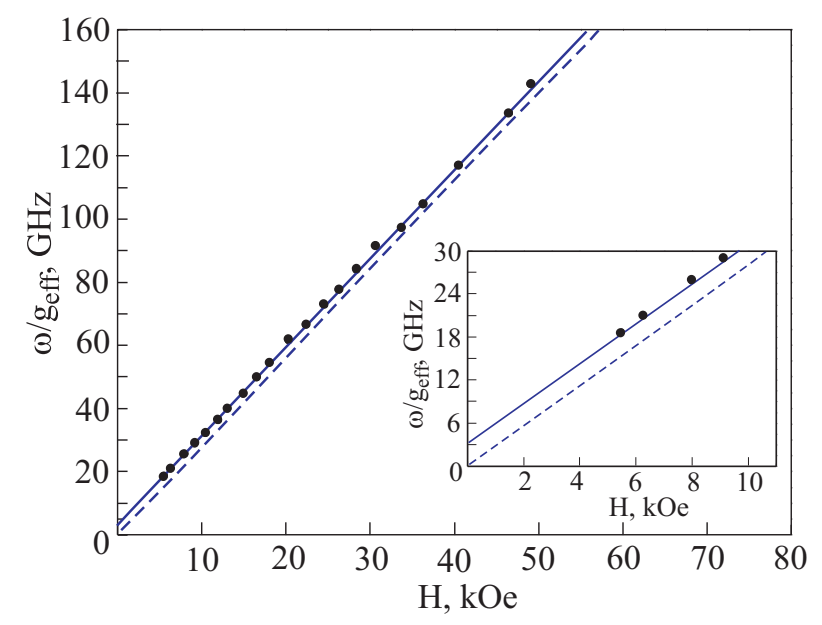

Puc. 3. The frequency-field dependence of ferrimagnetic resonance in $\mathrm{Cu}_{2} \mathrm{OSeO}_{3}, T=4.2 \mathrm{~K}$ : points — experiment, dashed line simple theory for an isotropic ferrimagnet, and solid line corresponds to Eq. (2 ). Inset: a magnified part of the frequency field dependence of the ferrimagnetic resonance. 
When the pumping power is going up, the resonance spectrum is transformed and some new features appear (see Fig. 4).

1. The spectrum consists of many components, very narrow absorption peaks appear in the resonance curve and the curve itself broadens. The number of peaks is dependent on the dimensions of the sample and on the structure of the hf variable magnetic field at the resonator location. As an example, Fig. 4 shows the resonance absorption curve measured at $f=\omega / \gamma_{\text {eff }}=21.32 \mathrm{GHz}$, which has four distinct maxima at the pumping power $P \sim 150 \mu \mathrm{W}$. The curve obtained at $\omega / \gamma_{\mathrm{eff}}=32.27 \mathrm{GHz}$ with the same microwave pumping has only two resonance absorption maxima.

2. As the high-frequency field power is raised to $P \sim 400 \mu \mathrm{W}$ and higher, an additional broad absorption line is observed in a magnetic field lower than the main resonance field (see Fig. 4, $\omega / \gamma_{\text {eff }}=25.8 \mathrm{GHz}$. and $\left.\omega / \gamma_{\text {eff }}=27.11 \mathrm{GHz}\right)$. When the temperature increases the threshold of the (hf) field rises. At $T=20 \mathrm{~K}$ it reaches $700 \mu \mathrm{W}$.

The effects of resonance absorption observed in the experiments on $\mathrm{Cu}_{2} \mathrm{OSeO}_{3}$ can be interpreted as follows. Real ferrimagnets possess magnetic anisotropy. In particular it is the magnetic crystalline anisotropy which includes the energy of magnetostrictive stresses. Because of this the effective magnetic crystalline anisotropy is significant when magnetization inhomogeneities are available over the crystal [4], and the resonance equation for the [111] direction becomes

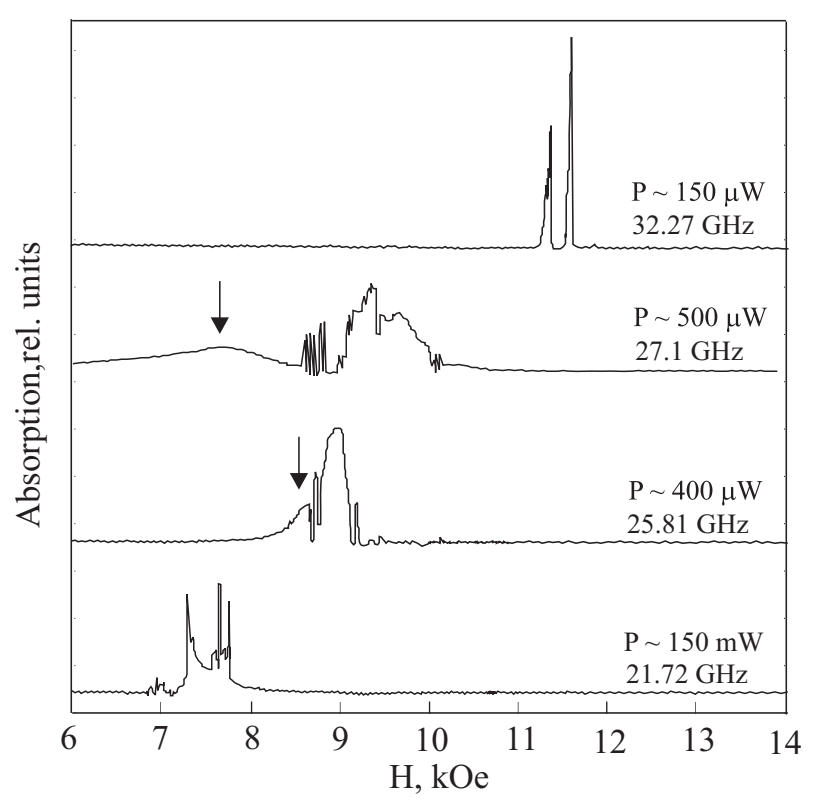

Puc. 4. The spectra of resonance absorption of the $\mathrm{Cu}_{2} \mathrm{OSeO}_{3}$ crystal in an external magnetic field along the [111] direction at different frequencies and microwave powers: The arrows show the parametric excitation. $T=4.2 \mathrm{~K}$.

$$
\omega=\gamma_{\mathrm{eff}} H_{0} \pm \frac{4}{3} \frac{K_{1}}{M_{S}},
$$

where $K_{1}$ is the magnetic anisotropy and $M_{S}$ is the saturation magnetization. Equation (2) can describe the frequency-field dependencies of $\mathrm{Cu}_{2} \mathrm{OSeO}_{3}$ quite adequately if the total magnetic anisotropy is $3 \mathrm{GHz}$. Thus existence of anisotropy leads to the gap in the ferromagnetic resonance spectrum of $\mathrm{Cu}_{2} \mathrm{OSeO}_{3}$.

Theoretically [5,6], the multicomponent structure (independent of pumping power) of resonance spectra taken on large and small samples can be explained assuming that small magnetic inhomogeneities (that are always available in ferrimagnets and couple uniform precession and spin waves to the wave vector $k \neq 0$ [7] produce a diversity of types of magnetization precession, which show up as absorption maxima. One of these types is a uniform precession, the rest are non-uniform, i.e. the amplitudes and phases of ac magnetization in the sample are dependent on its coordinates. The character of this dependence determines the type of precession. The resonance absorption maxima corresponding to uniform and nonuniform types of magnetization precession are shown in Fig. 4. For example, for two frequency $32.27 \mathrm{GHz}$ high field line corresponds to uniform precession, and low field line corresponds to nonuniform presession.

The intensity of excitation of different precession types depends on the structural similarity of the external variable field and the particular non-uniform type of magnetization — the closer the similarity the higher the intensity. This correlation determines the variation of absorption peaks and their intensities with the position of the sample in the resonator. Comparatively narrow resonance curves suggest that different types of precession are excited successively with varying $\omega$ or $H_{0}$, which was observed experimentally. Individual absorption peaks are best observed when the crystal is oriented along the easy magnetization axis. The presence of magnetic inhomogeneities leads [8] to excitation of degenerate uniform-precession spin waves with $k \neq 0$ which takes the energy from the uniform precession and transfers it over to the crystal lattice. This increases the magnetic losses (proportional to $\sqrt{M_{0}}$ ) and hence broadens the resonance curve. Thus, the maxima, their amplitudes and their broadening are determined by the dimensions of the sample, its magnetic inhomogeneities and the structure of the ac magnetic field at the sample location.

The frequency-field dependencies of resonance absorption were taken at the lowest power of (hf) field pumping $(\sim 100 \mu \mathrm{W})$.

The additional absorption and the saturation of the resonance line are nonlinear effects occurring when high frequency field $\mathbf{h}$ exceeds its threshold value for a ferrimagnet with small magnetic inhomogeneities. The nonlinear phenomena in ferrimagnets are caused by some instability making the oscillation amplitudes increase with time. This was detected for the first time in experiments on 
ferrimagnetic resonance in nickel ferrite in large amplitude (about $1 \mathrm{~kW}$ ) pumping fields [9]. The instability related phenomena (auto-oscillations, parametric excitation, autoparametric processes, etc.) are quite multiform in nonlinear systems. The occurrence of forced oscillations at frequencies different from the pumping ones is one of the fundamental properties of such systems.

The theory of nonlinear absorption was developed by Suhl [10]. According to [10], the increase in the microwave field amplitude up to the threshold magnitude entails a parametric increase in the amplitudes of the spin waves with frequency $\omega$ (ac field frequency) and wavelengths of $\sim 10^{-5} \mathrm{~cm}$. The amplitude of the spin waves with the frequency $\omega_{\mathrm{sp}}=\omega / 2$ as well as the wavelength tending to infinity are also increasing.

The first phenomenon leads to a broadening of the line of ferrimagnetic resonance with uniform precession. The other causes additional absorption in magnetic fields slightly lower than uniform resonant, $(0.5-0.9) H_{\text {res. }}$ The spin waves are excited at the expense of the energy of uniform-precession oscillations. This causes additional attenuation of the uniform precession seen as resonance line broadening. It must be noted that in our case the threshold of nonlinear effects is very low (lower then $1 \mathrm{~mW}$ ) in contrast to well known case of ferrites [9] The threshold field is [10].

$$
h_{\text {thres }}=\frac{\Delta H_{k} \Delta H_{0}}{4 \pi M_{0}},
$$

where $\Delta H_{k}$ is the half-width of the spin-wave resonance curve, $\Delta H_{0}$ is the half-width of the main resonance curve, and $4 \pi M_{0}$ is the demagnetization factor. The threshold field decreases with the width of the ferromagnetic resonance line, i.e. with decreasing magnetic losses at a low power level. The value of $h_{\text {thres }}-$ decreases considerably [10] when the field required to excite spin waves with $\omega_{\mathrm{sp}}=\omega / 2$ coincides with the main resonance (Fig. 4, $\left.\omega / \gamma_{\text {eff }}=25.81 \mathrm{GHz}, P \sim 400 \mu \mathrm{W}\right)$. The threshold power increases appreciably with temperature of the sample, particularly near $T_{c}$, because the magnetization of the ferrimagnet decreases.

It can be concluded that the observed additional absorption is caused by the parametric excitation of both short- and long- wavelength spin waves corresponding to the sizes of the available inhomogeneities.

\section{Conclusions}

The experimental results and their analysis can be summarized as follows.

The resonance properties of a new $\mathrm{Cu}_{2} \mathrm{OSeO}_{3}$ ferrimagnet have been investigated in a wide range of frequencies (17-142 GHz) at liquid helium temperature. The resonance data were used to plot the frequency-field dependence of the ferrimagnetic spectrum which is described within the model of an anisotropic two-sublattice ferrimagnet. The effective magnetic anisotropy corresponding to the gap in the spin wave spectrum has been estimated ( $3 \mathrm{GHz} \sim 1 \mathrm{kOe})$. It is found that with increasing of pumping level the spectrum obtains a multicomponent structure, which is due to a diversity of types of magnetization precessions.

As the amplitude of the ac magnetic field increases, an additional absorption is observed in the external magnetic field lower than the field of the main resonance. The detected additional absorption corresponds to the nonuniform nonlinear parametric resonance, connected with nonuniformity of magnetic structure in the ferrimagnetic $\mathrm{Cu}_{2} \mathrm{OSeO}_{3}$.

The threshold of nonlinear excitations in this ferrimagnet appears to be very low $(P<400 \mu \mathrm{W})$

The authors would like to thank Dr. V. Gnezdilov for fruitful discussions. Work at the EPFL was supported by the Swiss NSF and by the NCCR MaNEP.

1. E.A. Turov, Physical Properties of Magnetically Ordered Crystals (in Russian), AN SSSR Publ., Moscow (1963).

2. Jan-Willem G. Bos, Claire V. Colin, and Thomas T.M. Palstra, Phys. Rev. B78, 094416 (2008).

3. N.S. Akulov, Ferromagnetism, (in Russian), ONTI, Moscow-Leningrad (1939).

4. A.G. Gurevich, Ferrites at Ultrahigh Frequencies (in Russian), Phys.-mat. Literature Publish., Moscow (1960).

5. R.L. White and I.H. Solt, Phys. Rev. 104, 56 (1956).

6. J.E. Mercereau and R.P. Feynman, Phys. Rev, 104, 63 (1956).

7. E. Schlömann, J. Phys. Chem. Solids, 6, 242 (1958).

8. A.M. Clogston, H. Suhl, L.R. Walker, and P.W. Anderson, J. Phys. Chem. Solids, 1, 129 (1956).

9. N. Bloembergen, R.W. Damon, Phys. Rev. 85, 699 (1952).

10. H. Suhl, J. Phys. Chem. Solids 1, 209 (1957). 